\title{
Hacking the Human Bias in Robotics
}

\author{
AYANNA HOWARD and JASON BORENSTEIN, Georgia Institute of Technology, USA
}

\begin{abstract}
CCS Concepts: • Social and professional topics $\rightarrow$ Codes of ethics; ・ Computing methodologies $\rightarrow$ Intelligent agents; • Computer systems organization $\rightarrow$ Robotics;
\end{abstract}

Additional Key Words and Phrases: Artificial intelligence, bias, professional responsibility, robot ethics

ACM Reference format:

Ayanna Howard and Jason Borenstein. 2018. Hacking the Human Bias in Robotics. ACM Trans. Hum.-Robot Interact. 7, 1, Article 3 (May 2018), 3 pages.

https://doi.org/10.1145/3208974

Many of us, roboticists and those who collaborate with them, experience delight, excitement, and sometimes deep-seated, but rarely unvoiced, fears as we witness our robotic systems begin to impact human lives in countless ways. From automating driving to reshaping various facets of health care delivery, robotic systems are growing in their prevalence and intrusiveness into our daily lives. In combination with our siblings in the Artificial Intelligence (AI) community, scholars continue to predict a wide range of benefits from robotics and AI systems but also serious harms, including potential existential threats to humanity. Recognized pillars of science and engineering, including Elon Musk and the late great Stephen Hawking, have given voice to the apocalyptic kinds of fears that the public may have about an increasingly automated future. Whether these fears should be taken seriously is an issue that has divided scholars for awhile now, as illustrated by debates between Bill Joy [7] and Ray Kurzweil [8] at the beginning of the 21st century. On a different scale of granularity, a category of harms that users and others are more likely to experience on a day-to-day basis is from the various types of bias encoded in, or learned by, AI systems. This category of harms is especially troublesome in the world of physical robotics.

Nonembodied AI systems can obviously make decisions that have effects on human beings, such as a chatbot determining what to say in response to a customer's question on a company's helpline. Yet it will need to rely on an embodied entity (often a human) to have a direct impact on the physical world. Typically, a nonembodied AI agent provides input to humans who may then execute a physical action - whether those humans are making an employment decision to hire or fire or deciding on a health care intervention for a patient. By definition, it lacks the capability of acting on the world without assistance. Robots that have a physical form, on the other hand, can perform actions on their own. This can raise the ethical stakes in terms of the potential benefits and harms that may result from the technology. The benefits and harms that we are particularly concerned about here are related to bias.

Authors' addresses: A. Howard, Georgia Institute of Technology, School of Interactive Computing, Atlanta, GA, 30308, USA; email: ayanna.howard@gatech.edu; J. Borenstein, Georgia Institute of Technology, School of Public Policy, Atlanta, GA, 30332, USA; email: borenstein@gatech.edu.

Permission to make digital or hard copies of part or all of this work for personal or classroom use is granted without fee provided that copies are not made or distributed for profit or commercial advantage and that copies bear this notice and the full citation on the first page. Copyrights for third-party components of this work must be honored. For all other uses contact the Owner/Author.

2018 Copyright is held by the owner/author(s).

2573-9522/2018/05-ART3

https://doi.org/10.1145/3208974 
Bias is a pervasive feature of human life. It is intertwined, or used interchangeably, with many labels - stereotypes, implicit or subconsciously held beliefs, or close-mindedness. Bias can certainly have detrimental effects on human well-being, including in the realm of health care [4]; it is often connected to unfair practices, such as certain segments of society being blocked from meaningful access to affordable home loans or receiving disproportionately harsh criminal sentences. At times, these unfair practices may be tied to an AI system that is rendering decisions that guide human behavior (e.g., about who may be a good candidate for a home loan or who is likely to commit another crime), and the decisions by that system have been contaminated by human bias [5].

Given the breadth of effects that robots are expected to have on human life, the infusion of harmful forms of bias into the design and functioning of robotic technology must be scrutinized and hopefully halted. For example, a recent article in the ACM/IEEE International Conference on Human-Robot Interaction entitled "Robots and Racism" examined how research subjects react to a robot if they assume the robot has been "racialized"; the scenario was conducted under the guise of a shooter bias task [1]. The researchers illustrated that subjects' reaction time when deciding to shoot a robot differs depending on the "skin tone" of the robot. What this type of research does not do, however, is create algorithmic solutions on the system side once the potential problem has been identified. Studies are identifying that bias is occurring, but they often do not develop solutions to mitigate or halt it on a larger scale. This can be a shortcoming in much of the current robotics research.

As the media continues to sensationalize intelligent systems-such as chatbots [6], search engines [3], or facial recognition systems [9] - that display the same or similar biases as humans, we as members of the robotics community share in the sensationalism blame if we do not operationalize the problem and seek to develop concrete, implementable solutions. This is not to suggest that researchers should abandon the practice of conducting studies and publishing papers. Collecting data, clarifying concepts, and, more generally, building on the knowledge base are vitally important tasks. Yet, as part of the robotics community, we also have a professional responsibility to move beyond merely conducting studies that continue to confirm the presence of bias. We know that bias exists and that it is a significant and corrosive problem that infuses itself into countless human activities (e.g., Byrne [2]). Yet what is less clear is what to do about it, especially when viewing this problem through a robotic lens. For instance, AI techniques that seek to address the bias issue based on ensuring algorithm transparency, such as Explainable AI, may not work that well with physically embodied systems. If a self-driving car is about to run into a pedestrian crossing the road, will it help to tell the passengers inside the car what it's doing shortly before impact? And is it appropriate for the car to behave differently depending on how well those inside the car know the pedestrian? We need to push our collective thinking powers and expertise to develop real-world methods to mitigate the effects that bias may have, some that will borrow from our discoveries in AI but many others that are broader and more inclusive in order to address this bias conundrum.

Numerous companies are urgently pushing to get robotic technologies-including autonomous vehicles, robot caregivers, and drones-out into the world. We have to display equal if not more urgency toward efforts to address ethical and social issues related to robots, including how bias encoded in or learned by robots may affect consumers, workers, patients, and, more generally, the public. Perhaps a step in the right direction is encouraging more collaborations across the disciplines and drawing together a more inclusive set of perspectives into the robot design process. In either case, the robotics community needs to be an integral part of developing solutions. Maintaining the status quo of how technology is currently designed may result in decisions being made or possibly imposed on the robotics community by external entities. Even worse, the public may 
end up being harmed by robots because designers lacked sufficient moral imagination and were not proactive enough to address bias or other ethical concerns before deploying the technology. We do not want to repeat history in the sense of reacting after technological failure has already occurred and the public's trust has eroded; instead, we need to decide now how to build a better robotic future.

\section{REFERENCES}

[1] C. Bartneck, K. Yogeeswaran, Q. M. Ser, G. Woodward, R. Sparrow, S. Wang, and F. Eyssel. 2018. Robots and racism. In Proceedings of the 2018 ACM/IEEE International Conference on Human-Robot Interaction (HRI'18). ACM, Chicago, IL, 196-204.

[2] W. Byrne. 2018. Now is the time to act to end bias in AI. Fast Company (28 February 2018). Retrieved April 19, 2018 from https://www.fastcompany.com/40536485/now-is-the-time-to-act-to-stop-bias-in-ai.

[3] J. Carpenter. 2015. Google's algorithm shows prestigious job ads to men, but not to women. Independent (7 July 2015). Retrieved April 19, 2018 from http://www.independent.co.uk/life-style/gadgets-and-tech/news/ googlesalgorithm-shows-prestigious-job-ads-to-men-but-not-to-women-10372166.html.

[4] W. Hall, M. Chapman, K. M. Lee, Y. M. Merino, T. W. Thomas, and B. K. Payne. 2015. Implicit racial/ethnic bias among health care professionals and its influence on health care outcomes: A systematic review. American fournal of Public Health 105, 12, e60-e76.

[5] A. Howard and J. Borenstein. 2017. The ugly truth about ourselves and our robot creations: The problem of bias and social inequity. Science and Engineering Ethics fournal. DOI : http://dx.doi.org/10.1007/s11948-017-9975-2

[6] E. Hunt. 2016. Tay, Microsoft's AI chatbot, gets a crash course in racism from Twitter. The Guardian (24 March 2016). Retrieved April 19, 2018 from https://www.theguardian.com/technology/2016/mar/24/tay-microsofts-ai-chatbotgets-a-crash-course-in-racism-from-twitter.

[7] B. Joy. 2000. Why the future doesn't need us. Wired (1 April 2000). https://www.wired.com/2000/04/joy-2/.

[8] R. Kurzweil. 2001. Promise and Peril. Retrieved April 19, 2018 from http://www.kurzweilai.net/promise-and-peril.

[9] S. Lohr. 2018. Facial recognition is accurate, if you're a white guy. New York Times (9 February 2018). Retrieved April 19, 2018 from https://www.nytimes.com/2018/02/09/technology/facial-recognition-race-artificial-intelligence.html.

Received April 2018; accepted April 2018 